\title{
Endzeit als casus confessionis ${ }^{1}$
}

\author{
Anja Moritz
}

\begin{abstract}
„Denn jtzund ist die zeit fuerhanden / dauon Daniel vnd Johanes schreiben / das mann das Thier vnd sein Bilde mus anbeten / vnd jderman von jhm das malzeichen an seine stirn oder hende empfahen vnd nemen / so er anders keuffen vnd verkeuffen / sol / das ist / Man mus das INTERIM annemen / oder in der oeberacht sein / Christum verleugken / der sterben. // Denn solche List vnd Gewalt / sind noch nie so heuffig bey einander gewest / widder die Christen / sint der Christenheit anfang / als jtzund zu dieser zeit / da man nicht allein mit Fewr vnd Schwert / Sondern auch mit grosser List vnd behendigkeit des Wolredens / die Leute mit dem Antichrist sich zu vergleichen vnd $\mathrm{zu}$ vereinigen dringet vnnd zwinget. // Darumb ist dis die letzte vnd fehrlichste zeit." ${ }^{2}$
\end{abstract}

Der evangelische Theologe Nikolaus von Amsdorff verfasste diese Zeilen während der Belagerung der Stadt Magdeburg im Jahr 1551. Seine Diagnose wurzelte in einer extremen apokalyptischen Stimmung, die in der Mitte des 16. Jahrhunderts zum primären Deutungshorizont der Magdeburger Geistlichen werden sollte.

Jede Epoche ist von einer gewissen Endzeitstimmung gekennzeichnet, die die Geschichtsschreibung häufig als Krisenphänomen ${ }^{3}$ kategorisiert. Dass aber jene Endzeitstimmung nicht nur Ausdruck einer Krise, sondern zugleich auch deren Ursache sein konnte, wird dabei oft vernachlässigt. Gerade die lutherische Reformation ohne die gesteigerten eschatologischen Ängste zu denken, hieße einen Teil ihrer Ursprünge, ihrer Motivation und Wirkung zu verkennen. ${ }^{4}$

1 Der Aufsatz ist Bestandteil meines Dissertationsvorhabes zum politischen Denken in der ersten Hälfte des 16. Jahrhunderts.

2 Nikolaus von Amsdorff, Das itzund die rechte zeit sey / Christum vnd sein Wort zu bekennen / vnd auff keine andere zu warten sey. // Etliche sprueche / das man den Adiaphoristen nicht trawen noch gleuben sol. Magdeburg 1551, BIIr-v.

3 Zur Problematik der Krise als analytisches Konzept vgl. Hans-Michael Baumgartner, Institution und Krise, in: Gert Melville (Hg.), Institution und Geschichte. Theoretische Aspekte und mittelalterliche Befunde, Norm und Struktur, I. Köln/Weimar/Wien 1992, $97-114$, v. a. 105.

4 Hierzu u. a. Robin Bruce Barnes, Prophecy and Gnosis. Apocalypticism in the Wake of the Lutheran Reformation, Stanford 1988 sowie Volker Leppin, Antichrist und Jüngster Tag. Das Profil apokalyptischer Flugschriftenpublizistik im deutschen Luthertum 15481618 (Quellen und Forschungen zur Reformationsgeschichte, 69). Heidelberg 1999, der die Auffassung Hartmut Lehmanns, „etwa ab 1530 scheint dann dieses Thema [die Eschatologie - A.M.] aber insbesondere im Luthertum für einige Zeit nicht mehr besonders aktuell zu sein“, Hartmut Lehmann, Endzeiterwartungen im Luthertum im späten 16. und im frühen 17. Jahrhundert, in: Hans-Christoph Rublack (Hg.), Die 
Denn die apokalyptischen Texte der Bibel, wie die oben genannte Offenbarung des Johannes und das Buch Daniel, ermöglichten es den Zeitgenossen, ihre Situation in ihrer positiven und negativen Konnotation zu deuten und sie chronologisch in die Heilsgeschichte einzuordnen. In Zeiten verstärkter Unsicherheit boten jene Texte Orientierung durch die Verortung der Ereignisse im göttlichen Heilsplan. Eng verbunden mit dem apokalyptischen Denken war stets eine teleologische Geschichtsauffassung, die von einem zunehmenden Ordnungsverlust ausging und die Wiederherstellung der Ordnung in Aussicht stellte. 5 Was Ordnung konkret meinte, war bestimmt durch den jeweiligen Kontext. Dem Historiker ermöglicht eben jener Rekurs auf die Ordnung unter Umständen einen Einblick in die Diskussion von Normen - seien sie sozialer, religiöser, ethischer oder auch politischer Natur. Am Beispiel des Widerstandes der Magdeburger Geistlichen gegen das Interim soll die politische Dimension des apokalyptischen Denkens illustriert werden. Nach einer kurzen Skizzierung des historischen Rahmens im ersten Kapitel widmet sich der zweite Teil meines Beitrages der heilsgeschichtlichen Einordnung des Interimsgeschehens durch die Magdeburger. In einem dritten Abschnitt thematisiere ich die Auswirkungen des apokalyptischen auf das obrigkeitskritische Denken der evangelischen Geistlichen.

Bis zur Mitte des 16. Jahrhunderts war die noch junge lutherische Kirche zwar nicht de iure, aber de facto durch zahlreiche Anstände und Vereinbarungen mit dem altgläubigen Kaiser geduldet. Da es immer wieder zu Konflikten zwischen den konfessionellen Parteiungen kam, erachtete es Karl V. als seine kaiserliche Pflicht, den Reichsfrieden wieder herzustellen. Nachdem er 1547 auf militärischem Wege im Schmalkaldischen Krieg einen Sieg gegen das protestantische Verteidigungsbündnis erlangt hatte, versuchte er diesen Erfolg in der Religionsfrage zu wiederholen. Unabhängig von Papst und Konzil ließ er ein Edikt ausarbeiten und vom Reichstag beschließen, das einen Vergleich der beiden Konfessionen bis zu einer endgültigen Entscheidung durch ein freies und ge-

lutherische Konfessionalisierung in Deutschland (Schriften des Vereins für Reformationsgeschichte, 197). Heidelberg 1992, 545-554, hier 549 gründlich widerlegt.

5 Vgl. Jürgen Lebram, Art. Apokalyptik II: Altes Testament, in: Theologische Realenzyklopädie, Bd. III, Berlin 1978, 192-202, hier 192 sowie für das Mittelalter maßgeblich Tilman Struve, Endzeiterwartungen als Symptom politisch-sozialer Krisen im Mittelalter, in: Jan A. Aertsen/Martin Pickavé (Hgg.), Ende und Vollendung. Eschatologische Perspektiven im Mittelalter (Miscellanea Mediaevalia, 29). Berlin/New York 2002, $207-$ 226. 
meines Konzil vorsah. ${ }^{6}$ Diese zwischenzeitliche Regelung vom 15. Juni 1548

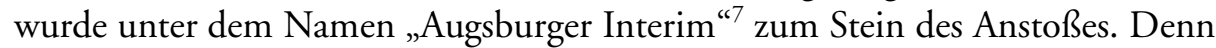
der angestrebte Vergleich war eine Ordnung altgläubigen Vorzeichens, die allein für die Protestanten gelten sollte und ihnen einzig Laienkelch und Priesterehe zugestand. Eine Annahme des Interims hätte nach damaliger Ansicht unweigerlich zur Aufgabe der Kernelemente lutherischer Lehre geführt. ${ }^{8}$ Während im Reich eine Widerstandsbewegung aufwallte, die der Kaiser zumindest in den süddeutschen Gegenden mit militärischer Gewalt unterdrücken konnte, ${ }^{9}$ arbeiteten auf Befehl des sächsischen Kurfürsten Moritz dessen Theologen und Räte eine Alternativordnung aus, die sowohl den altgläubigen Kaiser zufriedenstellen als auch die lutherische Lehre bewahren sollte. Diese Ordnung, die den Ständen auf dem Leipziger Landtag im Dezember 1548 vorlag, ${ }^{10}$ war allerdings so stark vom Kompromisscharakter geprägt, dass sie von Zeitgenossen als ein „Leipziger Interim" "11 betitelt und ebenso wie das Augsburger Interim abgelehnt wurde. Während das ganze Reich das kaiserliche Gebot einhielt,

6 Jüngst dazu Horst Rabe, Zur Interimspolitik Karls V., in: Luise Schorn-Schütte (Hg.), Das Interim 1548/50. Herrschaftskrise und Glaubenskonflikt (Schriften des Vereins für Reformationsgeschichte, 203). Heidelberg 2005, 127-146, der damit seine grundlegende Monographie Reichsbund und Interim. Die Verfassungs- und Religionspolitik Karls V. und der Reichstag von Augsburg 1547/1548. Köln/Wien 1971 anhand neuerer Quelleneditionen ergänzt bzw. korrigiert.

7 Der Text liegt ediert vor bei Joachim Mehlhausen (Hg.), Das Augsburger Interim. Nach den Reichstagsakten deutsch und lateinisch (Texte zur Geschichte der evangelischen Theologie, 3). Neukirchen-Vluyn 1962.

$8 \mathrm{Zu}$ den theologischen Aspekten des Augsburger Interims vgl. ausführlich Herbert Sowade, Das Augsburger Interim. Das kaiserliche Religionsgesetz von 1548 in seiner politischen und theologischen Relevanz für eine Einigung der Christen. Münster 1977, sowie zu deren Interpretation vgl. u.a. Irene Dingel, „Der rechten lehr zuwider“. Die Beurteilung des Interims in ausgewählten theologischen Reaktionen, in: Luise SchornSchütte (Hg.), Das Interim (wie Anm. 6), 292-311.

9 Hier sei nur auf die druckfrische Publikation der Beiträge einer Tagung des Vereins für Reformationsgeschichte hingewiesen: Luise Schorn-Schütte (Hg.), Das Interim (wie Anm. 6), die u. a. auch die wirkungsgeschichtlichen Aspekte des Interims berücksichtigt.

10 Hierzu die quellengesättigte Darstellung von Johannes Herrmann, Augsburg - Leipzig Passau. Das Leipziger Interim nach Akten des Landeshauptarchivs Dresden 1547-1552. Leipzig 1962 sowie Günther Wartenberg, Philipp Melanchthon und die sächsisch-albertinische Interimspolitik, Lutherjahrbuch 55 (1988) 60-82.

11 Matthias Flacius Illyricus gab die nicht beschlossene und somit nicht zur Publikation freigegebene Vorlage in den Druck, betitelte sie als das „Leipziger Interim“ und eröffnete damit die Auseinandersetzung mit den sächsischen Theologen. Diese von Flacius formulierte explizite Gleichsetzung des Augsburger Interims mit der Leipziger Landtagsvorlage wird samt der damit verbundenen polemischen Stoßrichtung auch heute noch unreflektiert verwendet, z. B. bei Alfred Kohler, Karl V. 1500-1558. Eine Biographie. München 1999, 326 oder Harm Klueting, Das konfessionelle Zeitalter 1525-1648. Stuttgart 1989, 132. 
weder gegen das Interim zu predigen noch zu schreiben, hielt sich die Altstadt Magdeburg als einzige Stadt nicht daran. Sie war bereits seit Juli 1547 in Folge der Niederlage im Schmalkaldischen Krieg in der Acht und weigerte sich trotz mehrfacher Aufforderungen, Moritz von Sachsen als ihren Schutzherren anzunehmen. $\mathrm{Zu}$ viel stand für die Einwohner auf dem Spiel: Sie befürchteten mit Recht den Verlust ihrer städtischen Freiheiten wie ihrer Religion. ${ }^{12}$ Aufgrund ihrer Widerständigkeit gegen alle Unterwerfungsversuche seitens des Kaisers wie des sächsischen Kurfürsten wurde Magdeburg zum Hort zahlreicher Flüchtlinge. Die lutherischen Geistlichen konnten hier gegen das Interim predigen und schreiben. Und dies taten sie mit einer Intensität, die der Stadt den Namen „Unseres Herrgotts Kanzlei“ einbrachte. ${ }^{13}$ Über ein Jahr wurde die Stadt durch die vom sächsischen Kurfürsten angeführten Truppen belagert, ${ }^{14}$ bis sich jener Moritz im Bündnis mit anderen Fürsten gegen den Kaiser wandte. Der so genannte Fürstenaufstand zwang den Kaiser im Sommer 1552 erfolgreich zu Friedensverhandlungen, zu denen auch die Aufhebung des Interims gehörte. ${ }^{15}$

Die zahlreichen Druckschriften, die in Magdeburg vor und während der Belagerung gedruckt wurden, sind geprägt von einem intensiven Endzeitbewusstsein. Ihre Verfasser, u. a. Nikolaus von Amsdorff $(1483-1565),{ }^{16}$ Nikolaus

12 Vgl. u.a. Dwaine Charles Brandt, The City of Magdeburg before and after the Reformation. A Study in the Process of Historical Change. Washington 1975 sowie Friedrich Hülße, Die Stadt Magdeburg im Kampfe für den Protestantismus während der Jahre 1547-1551. Halle 1892.

13 Siehe dazu ausführlich Thomas Kaufmann, Das Ende der Reformation. Magdeburgs „Herrgotts Kanzlei“ (1548-1551/52) (Beiträge zur historischen Theologie, 123). Tübingen 2003 sowie Nathan Baruch Rhein, The Chancery of God: Protestant Propaganda against the Empire. Magdeburg 1546-1551. Ann Arbor 2002.

14 Vgl. u. a. Simon Issleib, Magdeburgs Belagerung durch Moritz von Sachsen 1550-1551, in: Ders., Aufsätze und Beiträge zu Kurfürst Moritz von Sachsen (1877-1907), II. Leipzig 1989, 619-704.

15 Der Passauer Vertrag ist samt einigen Verhandlungsprotokollen ediert und mit einer ausführlichen Einleitung versehen durch Volker Hennig Drecoll (Hg.), Der Passauer Vertrag (1552). Einleitung und Edition (Arbeiten zur Kirchengeschichte, 79). Berlin 2000; vgl. auch Winfried Becker (Hg.), Der Passauer Vertrag von 1552: politische Entstehung, reichsrechtliche Bedeutung und konfessionsgeschichtliche Bewertung (Einzelarbeiten aus der Kirchengeschichte Bayerns, 80). Neustadt a.d. Aisch 2003.

16 Amsdorff wirkte bereits von 1524 bis 1541 als Superintendent in Magdeburg. Im Ergebnis des Schmalkaldischen Krieges verlor er sein Amt als Naumburger Bischof und war seit 1547 als Berater der kursächsischen Fürstensöhne tätig, bis er 1549 wegen des Interims nach Magdeburg floh; vgl. Biographisch-bibliographisches Kirchenlexikon, Bd. I, Hamm 1975, 152-154. 
Gallus $(1560-1570)^{17}$ und Matthias Flacius Illyricus $(1520-1575),{ }^{18}$ bezeichneten sich selbst als Exules Christi, da auch sie zu jenen gehörten, die aufgrund ihres Protestes gegen das Interim ihre Stelle aufgaben und Zuflucht in Magdeburg fanden. Die Deutung ihrer Situation als Zeichen des Endes, ihrer Zeit als Endzeit geschah in den verschiedensten Variationen, von denen hier nur einige wenige exemplarisch genannt seien.

Wurde auf arithmetische Beweisführungen nach Mk. 13 gänzlich verzichtet, lassen sich in den Druckschriften einige wenige Hinweise auf astrale Erscheinungen oder irdische Katastrophen, wie die apokalyptische Trias von Krieg, Pest und Teuerung, finden. Heuschreckenschwärme in Tirol, das Erscheinen dreier Sonnen und Monde oder das Bild des gefangenen sächsischen Kurfürsten Johann Friedrich am Firmament wurden als sichtbare Zeichen des Endes gedeutet. ${ }^{19}$ Im Zentrum der Gegenwartsanalyse stand jedoch der Antichrist, der sich als Werkzeug des Satans im Kampf gegen Gott und dessen Schöpfung über Christus erhebt und seine Macht auf Lügen und Gewalt aufbaut, mit denen er die Christen zum Abfall bewegt. Alles, was er ist und tut, ist er und tut er gegen Christus. Der Antichrist verkörpert das zerstörerische Chaos, dessen Dynamik sich gegen die von Gott gesetzte Schöpfungsordnung richtet. ${ }^{20}$ Mit der Offenbarung des Antichrists im Papsttum durch Martin Luther war die entscheidende Voraussetzung für die in 2. Thess. prophezeite Beseitigung des Antichrists erfüllt und somit das Ende der Geschichte in greifbare Nähe gerückt. An diesen bereits verbreiteten Topos konnten die Magdeburger Geistlichen anknüpfen, deren aktuelle Aufgabe nun darin bestand, die Werke des Antichrist in ihrer gegenwärtigen Umwelt zu identifizieren. In erster Linie bedeutete dies,

17 Nikolaus Gallus (dt. Hahn), der wie Amsdorff in Wittenberg studiert hatte, war bis zur Annahme des Augsburger Interims durch städtischen Rat 1548 in Regensburg als Diakon tätig. Für ein Jahr übernahm er in Wittenberg die Vertretung des Schlosspredigers Kaspar Cruciger, bis er 1549 nach Magdeburg ging und dort die Stelle des Superintendenten an St. Ulrich übernahm; vgl. Biographisch-bibliographisches Kirchenlexikon, Bd. II, Hamm 1990, 174.

18 Der über Venedig und Basel nach Wittenberg gelangte Illyrier war seit 1544 an der dortigen Universität als Professor für Hebräisch tätig, bis er im Zuge v. a. des Verhaltens der sächsischen Theologen gegenüber dem Interim die Stadt Richtung Magdeburg verließ; vgl. ebd., 43-48.

19 So geschildert im von Flacius Illyricus herausgegebenen Bericht des Braunschweiger Superintendenten Medler: Zeychen am himmel bey Braunschwig newlich gesehen / durch den superatendentem zu Braunschwig geschriben Mit einer vorrede, [Magdeburg] 1548.

20 Vgl. Sven S. Hartmann u. a., Art. Antichrist, in: Theologische Realenzyklopädie Bd. III, Berlin 1978, 20-50 sowie zu den verschiedensten Facetten der Figur des Antichrists Bernard McGinn, Antichrist: Two thousand Years of the Human Fascination with Evil. San Francisco 1994. 
den antichristlichen Charakter der beiden Religionsordnungen, des Augsburger Interims und der Leipziger Artikel, zu offenbaren.

Es ist „kund vnd offenbar / als die helle Sonne am Mittag“, so Nikolaus von Amsdorff, "das der Babst der rechte ware Antichrist ist / vnnd sein Roemischer hoff I des Antichrists reich ist / so hat das INTERIM seine muehe vnd arbeit verloren / vnd ist alles vergeblich vnnuetz vnd erlogen. " ${ }^{11}$ Obwohl der Kaiser das Augsburger Interim ohne das Einverständnis des Papstes hatte ausarbeiten lassen, ${ }^{22}$ stand es für die Magdeburger außer Frage, dass er als Diener des Antichrist nur in dessen Auftrag handeln konnte. Schon aus diesem Grunde war an eine Annahme des Interims nicht zu denken und schon gar nicht an die Umsetzung des Artikels 13, der die Unterordnung aller Christen und Bischöfe unter den Papst forderte. Die "Heimtücke“ des antichristlichen Vorgehens entlarvten die Geistlichen in der Forderung beider Religionsordnungen nach der Wiedereinführung der bereits beseitigten altgläubigen Zeremonien. Die sächsischen Theologen hatten nämlich in der Leipziger Landtagsvorlage den Zeremonien lediglich den Charakter von Mitteldingen, von Adiaphora, ${ }^{23}$ zugestanden, die man durchaus annehmen könne ohne die lutherische Lehre aufgeben zu müssen. Gerade die scheinbare Harmlosigkeit jener Zeremonien bildete aber in den Augen der Magdeburger das Einfallstor für eine gänzliche Rückführung der lutherischen Kirche in den Schoß der Altgläubigen. Schlimmer noch, hatte sich der Antichrist doch gerade jener großen Männer wie Philipp Melanchthon oder Johannes Bugenhagen als falscher Propheten bedient, die mit ihrer Leipziger Ordnung die Lehre verfälschten, Verwirrung unter den Gläubigen stifteten und sie letztlich zum Abfall trieben. Noch einmal Nikolaus von Amsdorff:

„Solche List vnd Gewalt / sind noch nie so heuffig bey einander gewest / widder die Christen / sint der Christenheit anfang / als jtzund zu dieser zeit / da man nicht allein mit Fewr vnd Schwert / Sondern auch mit grosser List vnd behendigkeit des Wolredens / die Leute mit dem Antichrist sich zu vergleichen vnd zu vereinigen dringet vnnd zwinget. / Darumb ist dis die letzte vnd fehrlichste zeit / Darinne wir vns wol moegen fuersehen / Das die Adiaphoristen vns mit jhrer falschen Disciplin vnd Zucht / nicht

21 Nikolaus von Amsdorff, Antwort / Glaub vnd Bekentnis auff das schoene vnd liebliche INTERIM. Niclasen von Amsdorffs des veriagten Bischoffs zur Naumburgk, 1548, DIIv.

22 Das Verhältnis zwischen Karl V. und Paul III. befand sich im Jahre 1547 auf dem Tiefpunkt, nachdem der Papst nicht nur das Konzil von Trient nach Bologna verlegt hatte und somit die konfessionellen Verständigungsbemühungen auf Eis gelegt wurden, sondern auch dessen Sohn angeblich im Auftrag des Mailänder kaiserlichen Statthalters ermordet wurde.

23 Der aus der Stoischen Ethik ins Christentum übernommene Begriff der Adiaphoria/

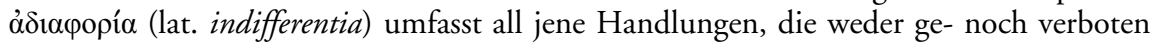
sind, die man als Erlaubte entweder tun oder lassen kann; vgl. hierzu den informativen Artikel von F. Lau, in: Religion in Geschichte und Gegenwart Bd. I, 3. Aufl., Tübingen 1957, 93-96. 
verfueren noch betriegen / Christum zuuerleugken / vnd den Antichrist anzubeten / das ist / seine malzeichen an vnsere stirn vnd hende zu nemen. ${ }^{\text {" } 24}$

Die Tatsache, dass beide Religionsordnungen durch die weltlichen Obrigkeiten angeordnet wurden, war Ausdruck der Vermischung des weltlichen mit dem geistlichen Regiment. So wie der Übergriff des Papsttums auf die weltliche Macht das Werk des Antichrist darstellte, deuteten die Magdeburger Geistlichen auch das Interim und den Leipziger Landtagsbeschluss als dessen Werk, das einzig auf die Zerstörung der Schöpfungsordnung ausgerichtet war. Denn diese war mit dem vom weltlichen Regiment geforderten Gehorsam in religiösen Belangen infragegestellt:

"Von dem Jnterim ist oeffentlich am tage“, so Nikolaus Gallus, „das dieselbe Kirchenordnung von den Obersten weltlichen Regenten herkompt / welchen Ampts halben nicht gebueret die Kirchen zu regiern / und das dieselben dem feinde Christi / dem Bapst zu Rom mit solcher ordnung dienen / und seiner Tyranney das wort Gottes und die Kirchen wiederumb unterwerffen". ${ }^{25}$

Der Papst als Antichrist forderte vom Kaiser das Interim. Somit war für Gallus sogar ein doppelter Regimentsübergriff gegeben: Der Papst regiert den Kaiser und der Kaiser die Gewissen. Und so konnte denn auch die mit der gewaltsamen Einführung des Interims einhergehende Vertreibung der Prediger ebenso wie die Belagerung der Stadt Magdeburg nur als letztes Aufbäumen des Antichrist vor seiner Vernichtung verstanden werden.

Alle Zeichen des Endes waren also durch Ordnungsverlust konstituiert: der Zerfall der vierten Monarchie nach Daniel, ${ }^{26}$ außer-ordentliche Geschehnisse in der Natur (Himmel oder Erde), der Abfall von der Lehre durch die eine in UnOrdnung gebrachte Lehre (durch Verfälschung nämlich) sowie die Zerstörung der göttlichen Schöpfungsordnung schlechthin durch die Vermengung der beiden Reiche. Der Antichrist setzte die Dynamik des Chaos gegen die statische Ordnung Gottes. Die Magdeburger Geistlichen diagnostizierten diesen Ordnungsverlust in ihrer Gegenwart anhand des endzeitlichen Vokabulars. Das Jüngste Gericht war ja bereits mit der Offenbarung des Antichrist durch Luther in eine greifbare Nähe gerückt. Zusätzlich identifizierten die Magdeburger in Augsburger Interim und Leipziger Landtagsbeschluss das Wirken des Antichrist, der sich durch die Vermengung der Reiche und den Abfall bzw. die Verfolgung der Christen für den Endkampf mit Christus wappnete. Hierzu bediente er sich seiner Werkzeuge, der weltlichen Obrigkeit in Gestalt des Kaisers und des

24 Nikolaus von Amsdorff, Das itzund (wie Anm. 2), BIIv.

25 Nikolaus Gallus, Eine DISPVTATION von Mitteldingen / vnd von den itzigen Verenderungen in Kirchen / die Christlich vnd wol geordnet sind / aus dem Latein verdeutscht. // Mit einer Vorrede, Magdeburg 1550, DIIr.

26 Dan. 2. 
sächsischen Kurfürsten sowie der falschen Propheten, den sächsischen Theologen. Die Theologen also, die eigentlich die Seelen behüten und den Glauben fördern sollten, brachten eben jene Gläubigen zum Abfall, indem sie sich dem Antichrist unterwarfen. Und die weltliche Obrigkeit, die eigentlich in der Funktion des Katechon den Antichrist aufhalten sollte, indem sie Kirche und Christen beschützte, machte sich zu dessen Diener. Der Angriff des Antichrist auf die wahre christliche Religion wurde von allen Seiten geführt und war demnach noch nie so gefährlich gewesen wie in der Mitte des 16. Jahrhunderts. Die Existenz der lutherischen Kirche stand auf dem Spiel. Und auch, nicht zu vergessen, die politischen und religiösen Freiheiten der Stadt Magdeburg, die seit dem Herbst 1550 von den Truppen des sächsischen Kurfürsten Moritz belagert wurde. Das Bewusstsein, am Endpunkt der Geschichte zu stehen, Zeuge des letzten Kampfes zwischen Gut und Böse zu sein, ein Bollwerk Christi, bestimmte nicht nur den Ton der Auseinandersetzung in den Druckschriften. Er engte auch den Handlungsspielraum der Magdeburger enorm ein, wie aber auch den der anderen Christen: „Ehr der HERR Christus stehet am Creutz / vnd wir mit jhm " ${ }^{27}$ Wer sich für die Magdeburger entschied, entschied sich für Christus. Wer sich gegen sie entschied und ihre Belagerung unterstützte, verriet Christus und machte sich zum Diener des Antichrist. Pro und contra waren die beiden Möglichkeiten, zwischen denen sich die Christen zu entscheiden hatten. Der casus confessionis war eingetreten: „Wenn jtzunder die zeit Christum vnd sein Wort zu bekennen nicht fuerhanden ist / So weis ich nicht wenn sie kommen sol", so Nikolaus von Amsdorff. ${ }^{28}$

\section{III}

Welche Konsequenzen für das Handeln ergaben sich nun aus einer derartigen Interpretation der Wirklichkeit als äußerste Gefahrensituation, als apokalyptischer Ernstfall? Im casus confessionis, wie er nach ausgiebiger Beweisführung der Magdeburger Geistlichen eingetreten war, galt es sich zu positionieren. Das Bekenntnis pro Christum war das Bekenntnis contra Antichristum. Aus der Interpretation dieser Lage heraus zogen die Magdeburger ihre Handlungsstrategien. Die Geistlichen waren als Hirten verantwortlich für die Bewahrung jeder einzelnen Seele, für deren Schutz vor der Versuchung durch das Böse, vor dem Zugriff des Antichrist. Arbeitete dieser mit den Mitteln des Chaos, mussten jene, um ihrer Hirtenfunktion gerecht zu werden, den Antichrist mit den

27 Prediger zu Magdeburg, Bekentnis Vnterricht vnd vermanung / der Pfarrhern vnd Prediger / der Christlichen Kirchen zu Magdeburgk, Magdeburg 1550, BIv [Ex.: Herzog-August-Bibliothek Wolfenbüttel: Yt5 Helmst. 40(21)].

28 Nikolaus von Amsdorff, Das itzund (wie Anm. 2), AIIr. 
Mitteln der Ordnung entgegentreten. Da diese Ordnung jedoch bereits im Zerfall begriffen war, bedurfte es ihrer Wiederherstellung. Diese erfolgte auf zweierlei Wegen: Zum einen durch die Entlarvung des antichristlichen Wesens der beiden Religionsordnungen und damit verbunden durch eine verstärkte Predigt des Evangeliums. Und zum anderen musste auf die ursprüngliche Schöpfungsordnung präskriptiv Bezug genommen werden, musste eingefordert werden, um die Ordnung wiederherzustellen. Hierzu bedienten sich die Prediger dem Deutungsmuster der Drei-Stände-Lehre. ${ }^{29}$

Bereits Luther, dessen Theologie von einem starken eschatologischen Gedanken geprägt war, reformulierte die Drei-Stände-Lehre als die göttlich gesetzte Ordnung gegen den Teufel und den Antichrist: „Drei Hierarchien nämlich hat Gott gegen den Teufel eingerichtet [...], und zwar die des Hauswesens, den Staat [...] und die Kirche." Der Papst stellte aufgrund seines antichristlichen Wesens gleichsam "das widerstaatliche Prinzip selbst“ dar. Die drei Stände hatten also nach Luthers Auffassung keine geringere Funktion, als das zerstörerische Wesen des Antichrist aufzuhalten, Wider-Stand zu sein. Die Unvergleichbarkeit des Papstes, des Anomos, die nur als eschatologisches Zeichen verstanden werden konnte, führte Luther zu „einer Art Ausnahmegesetzlichkeit“, so dass er hier von seiner Auffassung des passiven leiblichen Widerstandes abwich und einen aktiven leiblichen Widerstand jedes Einzelnen gegen den „Beerwolf“ und dessen Werkzeuge forderte:

„So, wenn der Papst einen Krieg erregen würde, so ist ihm Widerstand zu leisten, wie einem rasenden und besessenen Ungeheuer oder wahrhaft dem Arctolycos. [...] Und man soll sich nicht darum bekümmern, wenn es als Streiter für sich Fürsten, Könige, ja sogar Kaiser selber hat, überredet unter dem Titel Kirche. ${ }^{\text {“30 }}$

Jene beiden Thesen (66 und 68) der Zirkulardisputation konnten überraschenderweise nicht nur das aktive Notwehrrecht jedes Einzelnen rechtfertigen, sie waren zugleich auch Legitimation für den Widerstand gegen die Diener des Antichrist, also die weltlichen Obrigkeiten. Sprach 2. Thess. vom geistigen Widerstand mit dem Hauch des Mundes, so schloss Dan. 2 eine aktive Mit-

29 Dieses „Deutungsmuster sozialer Wirklichkeit“ sieht eine Dreiteilung der ständischen Gesellschaft vor in den status ecclesiasticus, status politicus und status oeconomicus. Alle drei Stände (auch als Hierarchien bezeichnet) waren aufgrund verschiedener Aufgabenbereiche voneinander geschieden, in Erfüllung dieser jedoch auch wiederum aufeinander angewiesen. Vgl. hierzu v. a. den Aufsatz von Luise Schorn-Schütte, Die Drei-StändeLehre im frühreformatorischen Umbruch, in: Bernd Moeller (Hg.), Die frühe Reformation in Deutschland als Umbruch (Schriften des Vereins für Reformationsgeschichte, 199). Gütersloh 1998, 435-461.

30 Zit. nach Rudolf Hermann, Luthers Zirkulardisputation über Matth. 19,21, Lutherjahrbuch 23 (1941) 35-93, der sich grundsätzlich mit dieser Disputation auseinandersetzt. 
arbeit des Menschen generell aus. Luther hatte 1539 mit diesen Thesen die Möglichkeit des aktiven leiblichen Widerstandes geschaffen. Elf Jahre später konnten sich die Magdeburger Geistlichen in ihrer Confessio darauf berufen.

Die Kollektivschrift der Magdeburger Geistlichen, „Bekenntnis, Unterricht und Vermahnung" aus dem Jahre $1550^{31}$, beginnt mit dem Verweis auf den Anbruch der letzten Zeit, auf die Offenbarung des Antichrist durch Luther und die Notwendigkeit des Bekenntnisses in den Zeiten der Verfolgung. Das Augenmerk nur auf die zentralen Sätze des Widerstandes der unteren Obrigkeit zu lenken, wie es die geistesgeschichtliche Forschung immer noch tut, ${ }^{32}$ vernachlässigt m. E. einen Großteil der Motivation, der das Bekenntnis seine Niederschrift verdankte. In diesem Sinne beschreibt auch das erste Kapitel der Confessio die drei Stände und ihre Aufgaben zum Erhalt der göttlichen Stiftung:

„Er [Gott] hatt auch alle drei Regiment / der Kirchen odder das Geistliche / Weltliche vnnd Hausregiment also von einnander gescheiden / das er einem jedern sein sonderlich ampt vnnd werck / auch seine sonderliche weyse zu straffen gegeben hatt. Vnd wiewol ehr nicht wil / das diese Regiment sollen in einander gemengt werden / so wil er doch / das eins dem andern dienen soll / vnnd samptlich in dieser entlichen meinung vnd werck vberein kommen / vnnd zusammen stimmen sollen / das ein jedes in seiner ordnung / vnnd nach seiner masse / rechte erkentnis vnd ehre Gottes / vnd er jenigen / so jhm zu regieren befohlen sindt / ewige seeligkeit foerderte vnd fortsetze." ${ }^{\text {“33 }}$

Folgt ein Regiment diesem göttlichen Gebot nicht und führt im Gegenteil die Schutzbefohlenen vom Glauben ab, wie es die Obrigkeit mit dem Interim praktizierte, „so entsetzen sie sich jhrer ehr selbst / das sie nicht mehr fuer Obrigkeit oder Eltern inn dem selben koennen gehalten werden ... Vnd werden nu aus Gottes ordnung ein ordnung des Teuffels welcher ordnung ein jeder nach seinem beruff mit gutem gewissen widderstehen kan vnd soll. ${ }^{34}$ Der teuflischen Unordnung widerstehen innerhalb der gottgesetzten Ordnung, nämlich des Berufs, ist bereits der äußerste aktive Widerstand, den die Magdeburger Geistlichen zu leisten in der Lage waren. Denn der Antichrist wird durch den Hauch des Mundes Christi getötet werden. Die weltliche Obrigkeit jedoch hatte das Schwert und konnte einen aktiven leiblichen Widerstand ausüben. Es war ihr Beruf, ihr Auftrag nach Röm. 13, die Kirche und die Christen zu schützen. Wenn also die höchste Obrigkeit diesen Auftrag nicht erfüllte und im Gegenteil zum Handlanger des

31 Siehe Anm. 27.

32 So u.a. Cynthia Grant Shoenberger, The Confession of Magdeburg and the Lutheran Doctrine of Resistance. Ann Arbor 1972; Robert von Friedeburg, Welche Wegscheide in die Neuzeit? Widerstandsrecht, "Gemeiner Mann“ und konfessioneller Landespatriotismus zwischen „Münster“ und „Magdeburg“, Historische Zeitschrift 270 (2000) 561 616 oder David M. Whitford, Tyranny and Resistance. The Magdeburg Confession and the Lutheran Tradition. Saint Louis 2001.

33 Prediger, Bekenntnis (wie Anm. 27), GIIv-GIIIr.

34 Ebd., GIVr. 
Antichrist wurde, „so kan vnnd sol eine Vntere / Gottfuerchtige Obrigkeit sich sampt den jhren / vber welche sie gesetzt ist / widder solchen vnrechten gewalt schuetzen / vnd auffhalten / auffs beste sie kan. ${ }^{35}$ Die von den Predigern angeführten vier Grade der ungerechten Gewalt mündeten im tyrannischen Wesen des Beerwolfs, der sich hoher weltlicher Obrigkeiten bediente, die Lehre Christi zu vertilgen. In diesem Fall hatten alle weltlichen Obrigkeiten die Pflicht, sowohl dem Beerwolf als auch seinen Werkzeugen zu wehren. Eben jene Situation war nach Ansicht der Prediger mit dem Interim eingetreten:

„Solchs aber geschicht jtzundt von vnsern Obern / das sie mit gewalt beyde in vns vnd in all vnsern nachkommen tilgen wollen das rechte erkentnis Gottes / ohn welches GOtt nicht kan geehret / auch kein Mensch seelig werden. Ja sie wollens noch fuer recht haben / vnd in aller Menschen hertzen pflantzen / falsche vnd GOtteslesterische Lehr / von Gott / des Antichrists vnd des Teuffels Reich auffrichten / vnd das Reich CHRisti zerstoeren ...".36

In der Interpretation der Magdeburger Geistlichen erwies sich die höchste weltliche Obrigkeit in einer Zeit akuter Bedrohung der Schöpfungsordnung, zu deren Schutz sie doch verordnet war, als nicht in der Lage, diese Aufgabe wahrzunehmen. Vielmehr machte sie sich mit dem Antichrist gemein und wurde dadurch selbst zur Gefahr. Dagegen nahmen sich die Prediger des geistigen Widerstandes gegen das Vordringen des Antichrist an, indem sie den aktiven Widerstand der unteren Obrigkeit legitimierten und mit der Offenbarung des Antichrist die Notwendigkeit des Erhaltes der Schöpfungsordnung in Form der drei Stände betonten. Jenes Beharren auf der göttlichen Ordnung kann jedoch nur in präskriptiver Hinsicht verstanden werden. Denn nach Luthers Offenbarungsleistung war deutlich geworden, dass einerseits der Antichrist schon seit geraumer Zeit auf Erden wandelte und andererseits durch dessen Vermengung der Ämter, die mit dem Interim einen Hochpunkt erreichte, die göttliche Ordnung längst zerstört oder zumindest geschwächt war. Umso notwendiger war ihr Widerstand und als umso erforderlicher erwies sich im Sinne der renovatio das Deutungsmuster der Drei-Stände-Lehre. Die Obrigkeit war damit längst nicht überflüssig geworden. Sie hatte sich jedoch den sie in ihre Schranken weisenden Predigern zu beugen, sich ihrem Strafamt zu unterwerfen. Nur durch die Wahrnehmung ihres Predigt- und Strafamtes konnten die Geistlichen in ihrem Stand gemeinsam mit dem status politicus und oeconomicus dem Antichrist standhalten. Dem zerstörerischen Chaos des Antichrist setzten die Magdeburger Geistlichen ein nachdrückliches Festhalten an der göttlichen Schöpfungsordnung entgegen. Die apokalyptische Verortung des Interims implizierte eine Deutungsmatrix, deren Schwarz-Weiß-Raster wieder-

35 Ebd., KIv.

36 Ebd., KIVv. 
um den Handlungsspielraum in Pro und Contra strukturierte. Jene Sicht auf die Umwelt musste entweder zur Unterwerfung unter den Antichrist führen oder zum Bekenntnis und einer radikalen Ablehnung des Interims. Die Magdeburger entschieden sich für letzteres. Und so konnte Nikolaus Gallus im Jahre 1552, dem Jahr der Aufhebung des Interims, bezugnehmend auf die Funktion des Katechon feststellen, dass der Antichrist durch die Prediger ,jtzt ein zeitlang auffgehalten worden / das er nit wider eingesessen " ${ }^{37}$

Apokalyptik ist also weder ein Rand- oder Unterschichtenphänomen, noch führt eschatologische Naherwartung zu Weltflucht. Im Gegenteil: Angesichts des bevorstehenden Jüngsten Gerichts ist jeder Christ aufgerufen zu verstärkter Verantwortung für sein eigenes Heil und eben auch für die Welt. Apokalyptik kann so auch als Sprache über Politik verstanden werden, als eine Möglichkeit, über politische und ethische Normen zu verhandeln. Die Warnung vor dem Antichrist und seiner übergroß werdenden Macht fügt sich nahtlos ein in die Diskussionen über die Figur des Tyrannen, fügt sich ein in die Bestrebungen, die Macht weltlicher Obrigkeiten zu beschränken, und sei es durch aristokratische Lösungen in Form der Drei-Stände-Lehre.

37 Nikolaus Gallus, Auff des Herrn D. Maiors verantwortung vnd Declaration der Leiptzigischen Proposition / wie gute werck zur seligkeit noetig sind / zum zeugnis seiner vnschuldt / das er mit der Leiptzigischen handlung nichts zu thun habe. // Antwort. Basel 1552, EIIr. 